\begin{tabular}{c|c|c|c|}
\cline { 2 - 3 } & REVISTA SABERES APUDEP & Volumen 4 Número 1 \\
\hline & ISSN L 2644-3805 & Enero-Junio 2020 \\
\hline
\end{tabular}

\title{
IMPACTO DEL COVID-19 EN LOS PUERTOS DE CONTENEDORES DE PANAMA
}

\author{
IMPACT OF COVID-19 ON PANAMA'S CONTAINER PORTS
}

\section{Francisco Valdés Góndola}

Universidad de Panamá, Centro Regional Universitario de Colón. Área Gestión Marítima. Panamá. igondola@yahoo.com http://orcid.org 0000-0002-3391-0145

\section{Yira Wong}

Universidad de Panamá, Centro Regional Universitario de Colón. Área Gestión Marítima. Panamá.carmenmanchong@gmal.com https://orcid.org/0000-0003-1693-1064

\section{RESUMEN}

La COVID-19 es la enfermedad infecciosa causada por el coronavirus que se ha descubierto más recientemente. Ambos eran desconocidos antes de que estallara el brote en Wuhan (China) en diciembre de 2019. Esta enfermedad comenzó a expandirse rápidamente por todo el mundo causando una crisis económica en las principales economías del mundo. La Covid-19 se evidencia en Panamá en el mes de marzo, esto trajo como consecuencia que el gobierno tomase la decisión de decretar una cuarentena absoluta en todo el país. Los puertos de contenedores de Panamá tomaron sus medidas sanitarias para seguir operando ya que estos son eje central del sistema logístico conjuntamente con el Canal de Panamá. Esta investigación tiene como propósito determinar el impacto económico, marítimo y logístico de los puertos de contenedores de Panamá durante los primeros seis meses de la pandemia en Panamá. Para el análisis documental se realizó una revisión de literatura mediante dos fases, una convergente que consistió en búsquedas en Google Académico utilizando como palabras clave: "Los puertos de contenedores de Panamá", sin restricciones de tiempo. La fase divergente consistió en la selección de los documentos cuyos títulos y resúmenes tuvieran mayor relación con concepciones y descripciones del movimiento de carga de contenerizada en los puertos de contenedores. Se documentan 6 puertos de contenedores Manzanillo International Terminal, Cristóbal, Colon Container Terminal, Balboa, PSA Panamá, Bocas Fruit.

PALABRAS CLAVE: Panamá, Puertos de Contenedores, impacto económico 


\begin{tabular}{|c|c|c|}
\cline { 2 - 3 } & REVISTA SABERES APUDEP & Volumen 4 Número 1 \\
\hline
\end{tabular}

\begin{abstract}
COVID-19 is the most recently discovered infectious disease caused by the coronavirus. Both were unknown before the outbreak broke out in Wuhan (China) in December 2019. This disease began to spread rapidly around the world causing an economic crisis in the main economies of the world. Covid-19 is evidenced in Panama in the month of March, this resulted in the government making the decision to decree an absolute quarantine throughout the country. The container ports of Panama took their sanitary measures to continue operating since they are the central axis of the logistics system together with the Panama Canal. The purpose of this research is to determine the economic, maritime and logistical impact of the container ports of Panama during the first six months of the pandemic in Panama. For the documentary analysis, a literature review was carried out through two phases, a convergent one that consisted of searches in Google Scholar using as keywords: "The container ports of Panama", without time restrictions. The divergent phase consisted of the selection of documents whose titles and summaries were most closely related to conceptions and descriptions of the movement of containerized cargo in container ports. 6 container ports are documented Manzanillo International Terminal, Cristóbal, Colon Container Terminal, Balboa, PSA Panama, Bocas Fruit Company.
\end{abstract}

KEYWORDS: Panama, Container Ports, economic impact

\title{
INTRODUCCIÓN
}

En diciembre de 2019, la Organización Mundial de la Salud (OMS), anuncia la existencia del síndrome respiratorio agudo grave (SARS-CoV-2), que provoca la enfermedad por coronavirus, covid-19. En marzo de 2020 se detectan en Panamá los primeros casos de covid-19, por lo cual el Gobierno nacional anuncia la emergencia nacional y una cuarentena total. La conectividad del país que antes era una ventaja comparativa se transforma en un punto de vulnerabilidad sanitaria.

En Panamá quedaron operando, El Canal de Panamá, algunas actividades dentro del Sistema Portuario Panameño como: la estiba de carga local para abastecimiento, el transbordo de mercancía, se regulo la pesca artesanal y las demás actividades se suspendieron como: el tráfico de pasajeros las actividades de cruceros. Todo esto para evitar el contagio y la propagación del virus. 


\begin{tabular}{|c|c|c|}
\cline { 2 - 3 } & REVISTA SABERES APUDEP & Volumen 4 Número 1 \\
\hline
\end{tabular}

Según la Autoridad Marítima de Panamá (AMP) el Sistema Portuario Panameño este compuesto de 45 puertos divididos en puertos de cabotaje los cuales son administrados por la AMP que suman 26 y puertos privados especializados que suman 19, de estos 6 son puertos de contenedores.

Los puertos de contenedores del sistema portuario panameño son de suma importancia para la economía del país ya que la industria portuaria en Panamá es la segunda de mayor importancia después del Canal de Panamá.

Por tal razón, analizaremos cuál es su desempeño en cuanto al movimiento de carga de transbordo y carga local, además de las operación, infraestructura, tecnología, almacenamiento, distribución y eficiencia con los que cuenta estos recintos portuarios.

Cómo ha afectado la pandemia COVID-19 al movimiento de carga.

Con el propósito de responder esta pregunta nos proponemos determinar el impacto económico, marítimo y logístico de los puertos de contenedores de Panamá durante los primeros seis meses de la pandemia en Panamá.

Este estudio tiene como objetivo hacer un análisis del movimiento de carga en los puertos de contenedores, localizados en Panamá, entre periodo comprendido de enero a junio del año 2019 y de enero a junio 2020.

\section{DESARROLLO}

\section{PUERTOS DE CONTENEDORES}

Rúa (2006) define el puerto como un lugar construido en la costa marítima o en las orillas de un río, dispuesto para que las embarcaciones puedan realizar las operaciones de carga y descarga de mercancías, embarque y desembarco de pasajeros, etc.

Por su parte Vélez y Salazar (2018), nos dicen que el puerto es una zona de tierra y agua dotada de unas obras y equipo que permitan principalmente la recepción de buques, para realizar operaciones de carga, descarga, y el almacenamiento, recepción y entrega de mercancías, así como el embarco y desembarco de pasajeros. Esta zona de tierra y agua incluye la infraestructura necesaria (obras de abrigo, muelles) así como la superestructura (construcciones fijas ubicadas sobre la 


\begin{tabular}{|c|c|c|}
\cline { 2 - 3 } & REVISTA SABERES APUDEP & Volumen 4 Número 1 \\
\hline
\end{tabular}

infraestructura tales como almacenes, silos, tinglados) y el equipamiento fijo y móvil necesario para la operativa (tuberías de transporte, grúas, tolva). Para acceder al puerto es necesaria la presencia de unas infraestructuras marítimas de acceso (canales de entrada, ayudas a la navegación) así como unas infraestructuras terrestres (carreteras, vías férreas)

La definición que da CEPAL (1990) muestra el carácter funcional de los puertos. Los puertos son interfaces entre los distintos modos de transporte y son típicamente centros de transporte combinado. En suma, son áreas multifuncionales, comerciales e industriales donde las mercancías no sólo están en tránsito, sino que también son manipuladas, manufacturadas y distribuidas.

Según el último informe de la Autoridad marítima de Panamá (2020) el Sistema Portuario Panameño se conforma de 45 puertos de los cuales 26 realizan actividades de cabotaje y estos son administrados por la Autoridad Marítima de Panamá y 19 son puertos especializados los cuales están en concesión privadas. De estos puertos privados 6 se especializan en carga contenerizada los cuales son los siguientes: Manzanillo International Terminal (MIT), Cristóbal Panamá Ports Company, Colon Container Terminal (CCT), ciudad de Colón, Panamá. Bocas Fruit ubicado en la provincia de Bocas del Toro, Panamá, estos cuatro puertos se encuentran en el litoral Atlántico. En el litoral Pacífico se encuentran los puertos de Balboa Panamá Ports Company y Port Singapur Authority Panamá International Terminal (PSA).

Para Arteaga \& K, (2017). Los puertos de contenedores son estructuras creadas a la orilla del mar dotado de gran profundidad para recibir barcos especializados en el transporte de contenedores y con equipo necesario para la manipulación de carga contenerizada.

Manzanillo International Terminal (MIT): El puerto de Manzanillo International Terminal (MIT) fue construido en la antigua base naval de los Estados Unidos en Coco Solo Sur. EI MIT está localizada en la costa Atlántica de Panamá, geográficamente en Latitud Norte 92144 43, Latitud Oste 79531.40 cerca de la entrada norte del Canal de Panamá. Esta interconectada con France Field, el área de bodegas más grande de la Zona Libre de Colón. Cruzando la Avenida Randolph, MIT ha desarrollado un parque logístico con cuatro sistemas de bodegas, área de almacenamiento de contenedores vacíos, acceso al ferrocarril y un área de actividades de valor agregado. 


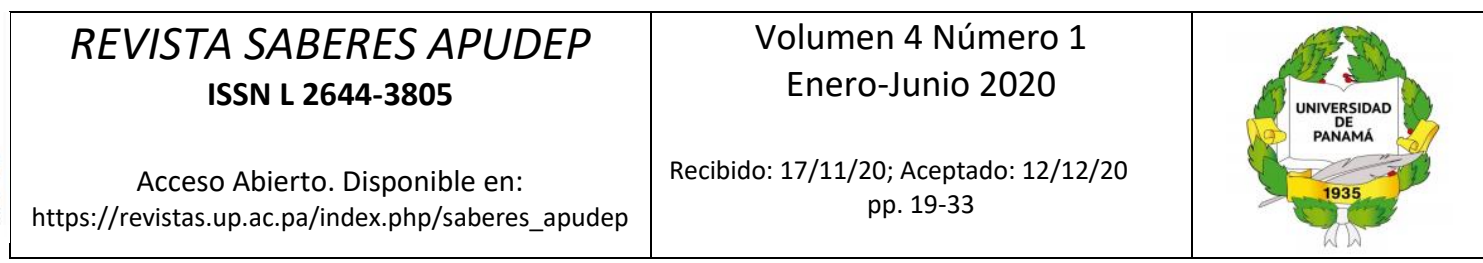

Las actividades portuarias que realiza el MIT son el movimiento de contenedores, carga rodante, cargas especiales, graneles secos, transbordo, carga general. Los equipos con los que cuenta la terminal son los siguientes: 11 grúas post-panamax, 8 grúas súper post-panamax, Grúas de patio 24 RTG (Rubber Tyred Gantry) 6 RMG (Rail Mounted Gantry, que son las 6 ASC), Top-picks, 27 marca Taylor, estiba a 5 de alto, Side-picks, 33 marca Taylor, estiba a 8 de alto.

En cuanto al movimiento de contenedores por unidad y por transbordo, la Tabla 1, muestra que el MIT ha tenido un comportamiento ascendente en ambos rubros. Par el año 2019 se operaron 639,486 contenedores que son muy buenos número mientras que en el 2020 en los primeros seis meses se reportó en operaciones 731,768 contenedores lo que significó un aumento del $14.4 \%$.

Esto significa que se movieron en el puerto en este periodo 92,282 contenedores más que el año 2019 lo que significa que el puerto ha mantenido buen ritmo en sus operaciones de contenedores pese a la pandemia mundial que se está dando.

Tabla 1. Movimiento de Contenedores Puerto de Manzanillo International Terminal enero-junio 2019-2020

\begin{tabular}{|c|c|c|c|c|}
\hline Año & Unidad & $\%$ & Transbordo & $\%$ \\
\hline 2019 & 639,486 & - & 544,663 & - \\
\hline 2020 & 731,768 & 14.4 & 657,819 & 20.7 \\
\hline
\end{tabular}

Fuente: Autoridad Marítima de Panamá

En cuanto al movimiento de contenedores en transbordo este también se dio de forma ascendente al registrar un aumento del $20.7 \%$ en el periodo en estudio 2020 . Esta es una de las principales operaciones que se dan en el puerto. Para este periodo se movieron 113.156 contenedores más que en el 2019.

Cristóbal Panamá Ports Company: El puerto de Cristóbal fue abierto para el comercio mundial el 24 de junio de 1904, localizados en el sector Atlántico en la ciudad de Colón. El primero de octubre de 1979 el puerto de Cristóbal revirtió al Gobierno Panameño al entrar en vigencia los tratados Torrijos-Carter, firmados en 1977 entre los Gobiernos de Estados Unidos de América y Panamá. 


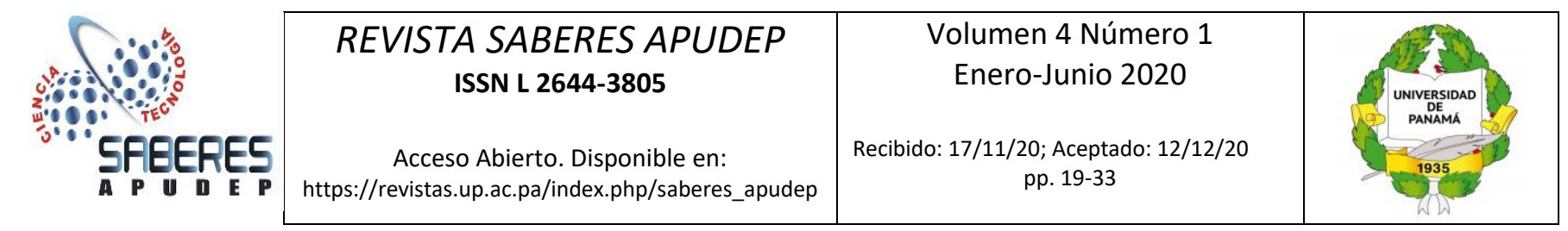

El 29 de julio de 1996, los puertos de Balboa y Cristóbal fueron concesionarios al consorcio Hutchinson Internacional Terminal de Hong Kong China, al ofrecer un pago fijo anual de 22.2 millones de dólares.

El puerto de Cristóbal se encuentra ubicado geográficamente Latitud norte $9^{\circ} 21^{\prime \prime} 11.83^{\prime \prime}$, Latitud oeste $79^{\circ} 54^{\prime 22}$.00". Se encuentra cercano a la Zona Libre de Colón, la Isla de Telfers y tiene carriles y carreteras de conexión a puntos a lo largo de Panamá incluyendo la ciudad de Panamá y el puerto de Balboa en el Pacífico. La localización estratégica de Cristóbal, cerca del caribe, América Latina, Norteamérica lo convierte en un lugar natural para el trasbordo.

Entre las actividades portuarias que realiza el puerto están las siguientes: el movimiento de contenedores, carga general, graneles, cruceros. Además, el puerto cuenta con 16 muelles para operaciones de contenedores, carga general, graneles y cruceros. Entre los equipos con los que cuenta el puerto están los siguientes: Grúas porticas: 9 grúas post-panamax, 4 panamax, 36 grúas de patio, 2 Reach Stackers, 14 Top-picks, 78 Chasis, 78 Truck, 8 Side-picks.

Al igual que el puerto de MIT el puerto de Cristóbal ha tenido un comportamiento ascendente en cuanto al movimiento de contenedores por unidad ya que se operaron 40,832 contenedores más en el 2020 , aumentando una taza porcentual de $13.9 \%$.

En el puerto de Cristóbal, Tabla 2, la carga de transbordo tuvo buen desempeño al aumentar en un $17.1 \%$ el movimiento del mismo. Con respecto al 2019 en el 2020 se operaron 47,764 contenedores en transbordo más que el año anterior.

Tabla 2. Movimiento de Contenedores. Puerto de Panamá Ports Cristóbal enero-junio 2019-2020

\begin{tabular}{|c|c|c|c|c|}
\hline Año & Unidad & $\%$ & Transbordo & $\%$ \\
\hline 2019 & 293,436 & - & 277,870 & - \\
\hline 2020 & 334,268 & 13.9 & 325,634 & 17.1 \\
\hline
\end{tabular}

Fuente: Autoridad Marítima de Panamá

Colon Container Terminal (CCT): es parte del Grupo Evergreen e inició operaciones en Panamá desde 1997. Desde 1994, el Grupo propuso la construcción de una 


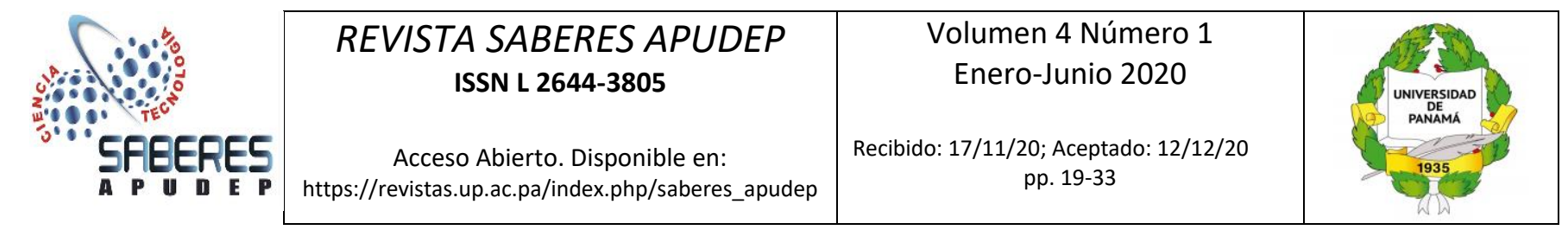

moderna terminal de contenedores aprovechando la posición geográfica de Panamá y los beneficios de conectividad del país y la amplia gama de servicios marítimos y logísticos. En 1995, un plan maestro para desarrollar una terminal de contenedores en Coco Solo Norte fue presentado al Gobierno panameño, y la nueva terminal nombrada CCT fue construida en el mismo lugar donde existía una antigua base naval de los Estados Unidos.

Colon Container Terminal está localizada geográficamente Latitud norte 92219 32, Latitud oeste 79525947 en Coco Solo Norte, provincia de Colón. Este puerto se ha convertido en un punto ideal para el trasbordo de carga entrando y saliendo hacia y desde la Zona Libre de Colón. Desde la entrada Atlántica del Canal de Panamá, CCT da servicio a los mercados regionales del Caribe, América del Norte, Central y Sur con embarques originados principalmente en el Lejano Oriente.

La actividad portuaria que realiza el puerto es la del movimiento de contenedores, los cuales posee 4 muelles para realizar estas operaciones. Entre los equipos que cuenta el muelle están los siguientes: 5 grúas Panamax, 3 Post-Panamax, 3 Super PostPanamax, 30 RTGs, 3 Reach Stackers, 11 Top-picks. 93 chasis, 78 Truck, 14 Forklifth

En el puerto de Colon Container Terminal CCT disminuyó el número de contenedores manejados en el periodo de enero a junio del 2020. La operación de contenedores disminuyo en un $-4.35 \%$ debido a que gran parte de los clientes son locales y de Zona Libre de Colón, pero en los últimos meses mayo y junio este recinto se está recuperando.

En cuanto a la carga de transbordo, Tabla 3, la misma disminuyo en $-1-25 \%$ debido a que varios mercados del caribe dejaron de percibir carga por los efectos de la pandemia. En este sentido el puerto se ha ido recuperando paulatinamente en los meses de mayo y junio

Tabla 3. Movimiento de Contenedores. Puerto de Colon Container Terminal enero-junio 2019-2020

\begin{tabular}{|c|c|c|c|c|}
\hline Año & Unidad & $\%$ & Transbordo & $\%$ \\
\hline 2019 & 205,709 & - & 159,470 & - \\
\hline 2020 & 196,749 & -4.35 & 157,475 & -1.25 \\
\hline
\end{tabular}

Fuente: Autoridad Marítima de Panamá 


\begin{tabular}{|c|c|c|}
\cline { 2 - 3 } & REVISTA SABERES APUDEP & Volumen 4 Número 1 \\
\hline
\end{tabular}

Balboa Panamá Ports Company: fue inaugurado en 1909 luego de recibir su nombre actual, el cual se llamó Ancón Port. Fue restaurado por los estadounidenses al comienzo de la construcción del Canal, lo que anteriormente fue una antigua zona de atraque de barcos franceses. Balboa actualmente está en concesión y es administrado por Panamá Port Company. El puerto es uno de los puertos actualmente más productivos de Latinoamérica y con importantes clientes como son: Maersk y Mediterranean Shipping Company.

Balboa posee una ubicación geográfica Latitud norte $8^{\circ} 57^{\prime \prime} 38.47^{\prime \prime}$ y Latitud oeste 79³3'54.38" ideal para crecer como un centro de distribución de mercancías conectando los principales servicios de línea desde el Lejano Oriente y América del Norte, hacia la Costa Oeste de América del Sur, Central y el Caribe.

Con un total de 30 hectáreas dedicadas al almacenamiento de contenedores y 5 muelles para barcos portacontenedores, Balboa opera con 25 grúas porticas (10 Post Panamax, 8 Panamax y 7 Super Post Panamax) y 47 RTGs. Las operaciones de trasbordo de carga contenerizada representan el $92.8 \%$ del total de movimiento de contenedores, mientras que el resto está dirigido al mercado local. Un acceso directo con el ferrocarril permite el trasbordo de contenedores con destino a los puertos de Colón. Balboa también tiene capacidad de recibir y despachar graneles secos y líquidos, así como carga especializada, disponiendo de un total de 3,612 conexiones para refrigerados.

El puerto de balboa es uno de los puertos con mayor movimiento en el Pacifico panameño en cuanto al movimiento por unidad y transbordo de contenedores. En los primeros seis meses del año 2020, Tabla 4, el puerto operó 537,397 unidades de contenedores aumentando su producción en igual número de meses en el año 2019 en $6.2 \%$.

Tabla 4. Movimiento de Contenedores. Puerto de Colon Container Terminal enero-junio 2019-2020

\begin{tabular}{|c|c|c|c|c|}
\hline Año & Unidad & $\%$ & Transbordo & $\%$ \\
\hline 2019 & 505,888 & - & 438,049 & - \\
\hline 2020 & 537,397 & 6.2 & 485,890 & 10.8 \\
\hline
\end{tabular}

Fuente: Autoridad Marítima de Panamá 


\begin{tabular}{|c|c|c|}
\cline { 2 - 3 } & REVISTA SABERES APUDEP & Volumen 4 Número 1 \\
\hline
\end{tabular}

En cuanto al movimiento de transbordo el puerto tuvo un excelente desempeño operando 485,890 contenedores en este tipo de operación en los primeros 6 meses del año 2020 teniendo un aumento del 10.8\% con respecto al 2019.

Port Singapur Authority Panamá International Terminal (PSA): es un importante puerto construido por PSA Internacional, situado en la antigua base naval de Rodman, en la entrada Pacifico del Canal de Panamá. El mismo representa una importante escala para las líneas de trasporte marítimo de manipulación de contenedores y carga.

La concesión para la operación del puerto fue otorgada a través del Contrato Ley 36 del 19 de junio de 2008 celebrado entre el Estado panameño y PSA Panamá. PSA Panamá opera desde el año 2010 y es subsidiaria de PSA International, uno de los grupos portuarios líderes a nivel global con participación en unas 40 terminales en 16 países en Asia, Europa y las Américas y una fuerza laboral de más de 30,000 personas.

La terminal inició operaciones en diciembre de 2010 con la recepción de productos de hierro y otros suministros para la expansión del Canal de Panamá. Sus operaciones de contenedores iniciaron formalmente en el año 2012.

Su cercanía al Canal de Panamá permite un fácil acceso a la principal ruta intermodal de las Américas. Con su capacidad actual, la terminal de contenedores de PSA Panamá, atiende un estimado de 400 atraques y mueve 250,000 TEU por año.

El puerto se dedica a exclusivamente al movimiento de contenedores, cuenta con 3 muelles para operaciones de carga. Además, el puerto cuenta con los siguientes equipos: 11 grúas post-panamax, 9 Reach Stackers, 12 Top-picks.

PSA Panamá International, Tabla 5, ha venido teniendo un buen desempeño en los últimos tres años. En el estudio que estamos realizando en los primeros seis meses del año 2020 el puerto tuvo una operación de contenedores en unidad de 345,269 lo que representó un aumento del $33.3 \%$ es decir, manejo 86,359 contenedores más que en los primeros seis meses del 2019. 


\begin{tabular}{|c|c|c|}
\cline { 2 - 3 } & REVISTA SABERES APUDEP & Volumen 4 Número 1 \\
\hline
\end{tabular}

Tabla 5. Movimiento de Contenedores. Puerto de PSA Panamá International enero-junio 2019-2020

\begin{tabular}{|c|c|c|c|c|}
\hline Año & Unidad & $\%$ & Transbordo & $\%$ \\
\hline 2019 & 258,910 & - & 245,930 & - \\
\hline 2020 & 345,269 & 33.3 & 345,269 & 40.3 \\
\hline
\end{tabular}

Fuente: Autoridad Marítima de Panamá

En cuanto a las operaciones de transbordo estas aumentaron en $40.3 \%$ operando 99,339 contenedores más que el año pasado. Pese a la crisis económica por la pandemia se espera que el puerto llegue al medio millón de contenedores transbordado.

Bocas Fruit CO.: El puerto de Almirante se encuentra en la provincia de Bocas del Toro en el noreste de Panamá. Fue fundado en 1908 y está cerca de la frontera con Costa Rica, ha sido históricamente una puerta de entrada para la exportación de frutas, con especial atención a la exportación de bananas.

Administrada por la sucursal de Chiquita en Panamá, la terminal Bocas Fruit Company ofrece 312 metros de muelle con una profundidad de 11,00 metros. Está equipada con dos grúas portuarias móviles.

El patio de la terminal tiene una capacidad de 1.400 contenedores de 40 pies refrigerados (las bananas se suelen enviar en contenedores de 40 pies) y más de 500 conexiones reefer.

Tabla 6, Básicamente el puerto de Bocas Fruit opera contenedores en unidades que son para la exportación e importación. El puerto tuvo un buen crecimiento en los primeros seis meses siendo este de un $70 \%$ en el periodo que estamos estudiando. 


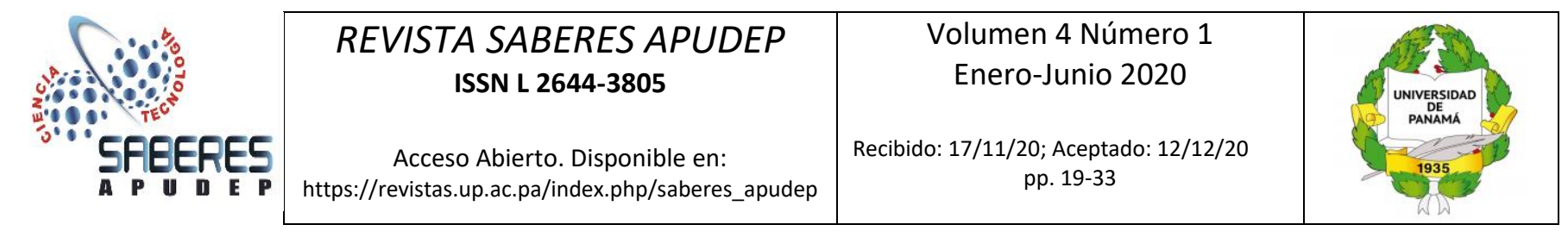

Tabla 6. Movimiento de Contenedores. Puerto de Bocas Fruit. enero-junio 20192020

\begin{tabular}{|c|c|c|c|c|}
\hline Año & Unidad & $\%$ & Transbordo & $\%$ \\
\hline 2019 & 16,958 & - & - & - \\
\hline 2020 & 28,837 & 70 & - & - \\
\hline
\end{tabular}

Fuente: Autoridad Marítima de Panamá

Pese a la pandemia que viene azotando la economía panameña los puertos de contenedores han experimentado un crecimiento en cuanto a unidades operadas y al segmento de transbordo

Todas las terminales portuarias se han mantenido operando $24 / 7$ en medio de la pandemia del coronavirus Covid-19, jugando un papel preponderante para el abastecimiento de alimentos, insumos, y otros productos básicos a nivel local y regional.

El último informe de la Autoridad Marítima de Panamá (AMP), actualizado al pasado 10 de julio de 2020, muestra que los puertos de contenedores este año experimentaron un crecimiento exponencial en comparación a igual periodo de 2019.

De acuerdo con las estadísticas, en los puertos de contenedores panameños se percibió un incremento de $13.2 \%$ en el movimiento de carga. Estos puertos se caracterizan por conectarse con los principales servicios de línea a nivel mundial.

Durante los primeros seis meses los puertos que más se destacaron fueron Manzanillo International Terminal en el Atlántico operando 731,768 unidades de contenedores y Balboa en el Pacífico operando 537,397 unidades de contenedores.

En cuanto al movimiento de transbordo en los puertos de contenedores en Panamá este creció $17.8 \%$ debido a las exportaciones de productos frescos desde Sudamérica hacia Estados Unidos y Europa, al igual que la evacuación de contenedores vacíos para reabastecimiento.

El puerto de Manzanillo International Terminal (MIT), es el puerto que más se destacó con un aumento en sus operaciones de transbordo de $20.7 \%$ seguido del puerto de Cristóbal con un aumento de $17.7 \%$. Mientras que en el Pacífico PSA tuvo un importante incremento del $40.3 \%$ en sus operaciones de transbordo 


\begin{tabular}{|c|c|}
\hline $\begin{array}{c}\text { REVISTA SABERES APUDEP } \\
\text { ISSN L 2644-3805 } \\
\text { Acceso Abierto. Disponible en: } \\
\text { https://revistas.up.ac.pa/index.php/saberes_apudep }\end{array}$ & $\begin{array}{c}\text { Volumen } 4 \text { Número } 1 \\
\text { Enero-Junio } 2020 \\
\text { Recibido: 17/11/20; Aceptado: 12/12/20 } \\
\text { pp. 19-33 }\end{array}$ \\
\hline
\end{tabular}

Tabla 7, se muestra que el único puerto que mostro cifras negativas fue el puerto de Colon Container Terminal esto debido a que gran parte de sus clientes locales son de la Zona Libre de Colon, Venezuela, Colombia y Republica Dominicana.

Tabla 7. Movimiento de contenedores en los puertos de carga contenerizada por unidad y transbordo periodo enero a junio $2019-2020$

\begin{tabular}{|c|c|c|c|c|c|c|}
\hline & \multicolumn{2}{|c|}{ AÑO 2019} & \multicolumn{2}{|c|}{ AÑO 2020} & $\%$ & \\
\hline $\begin{array}{l}\text { PUERT } \\
\text { OS }\end{array}$ & $\begin{array}{l}\text { UNIDA } \\
\text { D }\end{array}$ & $\begin{array}{c}\text { TRANSBO } \\
\text { RDO }\end{array}$ & $\begin{array}{l}\text { UNIDA } \\
\text { D }\end{array}$ & $\begin{array}{c}\text { TRANSBO } \\
\text { RDO }\end{array}$ & UNIDAD & $\begin{array}{c}\text { TRANSBO } \\
\text { RDO }\end{array}$ \\
\hline MIT & $\begin{array}{r}639,48 \\
6 \\
\end{array}$ & 544,663 & $\begin{array}{r}731,76 \\
8 \\
\end{array}$ & 657,819 & 14.4 & 20.7 \\
\hline $\begin{array}{c}\text { Cristób } \\
\text { al }\end{array}$ & $\begin{array}{r}293,43 \\
6 \\
\end{array}$ & 277,870 & $\begin{array}{r}334,26 \\
8 \\
\end{array}$ & 325,634 & 13.9 & 17.1 \\
\hline Balboa & $\begin{array}{r}505,88 \\
8 \\
\end{array}$ & 438,094 & $\begin{array}{r}537,39 \\
7\end{array}$ & 485,890 & 6.2 & 10.8 \\
\hline CCT & $\begin{array}{r}205,70 \\
9 \\
\end{array}$ & 159,470 & $\begin{array}{r}196,74 \\
9 \\
\end{array}$ & 157,475 & -4.35 & -1.25 \\
\hline PSA & $\begin{array}{r}258,91 \\
0 \\
\end{array}$ & 245,930 & $\begin{array}{r}345,26 \\
9 \\
\end{array}$ & 336,362 & 33.3 & 40.3 \\
\hline $\begin{array}{c}\text { Bocas } \\
\text { Fruit }\end{array}$ & 16,958 & - & 28,837 & - & 70 & - \\
\hline TOTAL & $\begin{array}{r}1,920,3 \\
87\end{array}$ & $1,666,027$ & $\begin{array}{r}2,174,2 \\
88\end{array}$ & $1,963,180$ & 13.2 & 16.2 \\
\hline
\end{tabular}

Fuente: Autoridad Marítima de Panamá

\section{CONCLUSIÓN}

Al terminar el estudio Impacto del COVID-19 en los puertos de contenedores de Panamá llegamos a la conclusión de que el movimiento de carga en su conjunto no ha sido afectado ni las operaciones de transbordo que estos realizan.

De acuerdo con las estadísticas de la AMP, de enero a junio de 2020, los puertos de contenedores de Panamá movieron 2,174,288 de contenedores, lo que significó un aumento de $13.2 \%$ con respecto a igual periodo en el 2019 , siendo el MIT la terminal que más contenedores opero con un $34 \%$ y Balboa con un $25 \%$

En cuanto al movimiento de transbordo este creció durante los primeros seis meses del 2020 en un 17.8\% destacándose el puerto de Manzanillo International Terminal con un aumento en sus operaciones de $33 \%$ y el puerto de Balboa con un aumento 


\begin{tabular}{|c|c|c|}
\cline { 2 - 3 } & REVISTA SABERES APUDEP & Volumen 4 Número 1 \\
\hline
\end{tabular}

del $25 \%$. el crecimiento del movimiento de transbordo fue apalancado, principalmente, por la temporada alta de exportaciones de productos frescos desde Sudamérica al hemisferio norte. Esto a través de la utilización de reefers y la evacuación masiva de equipos vacíos con el propósito de cumplir la demanda del servicio WCSA (red de exportaciones de productos frescos desde la Costa Oeste de Sudamérica a Estados Unidos y Europa).

El transbordo de contenedores constituye el negocio más rentable para la economía panameña después del Canal de Panamá. La economía del país se impacta positivamente, pues con ello no solo se favorecen los puertos, sino también el sector transporte por carretera que realiza los movimientos de transbordo desde un puerto a otro, así como el ferrocarril. Con esto queda comprobado que Panamá es un centro de transbordo importante en la región, ofrece una conectividad sin igual, conectando puertos de categoría mundial en el Atlántico y en el Pacifico en menos de $80 \mathrm{~km}$. Y que, además, es el único país que ofrece tres modalidades de trasbordo: barco a barco, por carretera y por tren.

La Autoridad Marítima de Panamá y las autoridades de los puertos de contenedores han estado trabajando sin parar desde el inicio de la crisis del covid-19 para asegurar el funcionamiento de la cadena de suministro local e internacional.

El puerto de Bocas Fruit ubicado en la provincia de Bocas del Toro es el único puerto que la Autoridad Marítima clasifica como puerto de contenedores, pero no realiza operaciones de transbordo.

El sector logístico y el sector marítimo han estado trabajando sin parar desde el inicio de la crisis del COVID-19 para asegurar el funcionamiento de la cadena de suministro local e internacional. El flujo continuo de mercancías, a través de nuestro territorio, representa hoy miles de trabajos a través del encadenamiento de actividades que, relacionadas entre sí, aseguran el tránsito seguro y a tiempo de la carga que pasa por nuestro territorio. Este encadenamiento de actividades tiene el potencial de crecer de la mano de actividades de valor agregado que ya se están dando y que solo necesitan desarrollar todo su potencial. 


\begin{tabular}{|c|c|c|}
\cline { 2 - 3 } & REVISTA SABERES APUDEP & Volumen 4 Número 1 \\
\hline
\end{tabular}

\section{REFERENCIAS BIBLIOGRÁFICAS}

Alvino, I (2014). Análisis del Sistemático de los Puertos Marítimos de Europa y Panamá. Tesis Doctoral. Universidad Cooperativa. Colombia.

Arteaga, Q., \& K, L. (2017). Mejoramiento y ampliación del puerto de aguas profundas en la ciudad de Manta y su impacto económico en el desarrollo local. Tesis Doctoral. Universidad de Guayaquil. Ecuador.

Autoridad Marítima de Panamá (2020). https://amp.gob.pa/estadistica/

CEPAL. (1990). Informe del seminario-taller sobre el análisis de la contaminación de la Refinería Estatal Esmeraldas en los ríos Esmeraldas y Teaone. Esmeraladas: CEPAL.

Colon Container Terminal. (2020) http://www.cct-pa.com/

Giraldez, J (2018). La Geografía y su Impacto en Logística Portuaria: Caso Puerto de Balboa, República de Panamá. Tesis de Maestría. Universidad de Panamá. Panamá

Hutchinson Ports - PPC. (2020). Conoce PPC. (https://www.ppc.com.pa/sitio/\#!/es/meetppc)

Ibáñez, I (2014). Análisis de la Industria Marítima Auxiliar. Tesis Doctoral. Universidad de Cataluña. España-

Ledezma, A (2020). Modelos de Evolución del COVID-19 en Panamá. Tesis Doctoral. Universidad Carlos III. Madrid España

Manzanillo International Terminal. (2020). Operaciones. (http://www.mitpan.com/operaciones/)

Ministerio de Salud (2020). http://www.minsa.gob.pa/

Mora, J. (2018). Análisis Comparativo entre el Sistema Portuario Panameño y el Sistema portuario colombiano. Tesis Doctoral. Universidad Católica. Colombia.

PSA Panamá. (2020). Nuestras Operaciones. (http://www.psa.com.pa /index.php?option=com_content\&view=article\&id=1\&ltemid=112\&lang=es)

Rúa, C. (2006). Los Puertos en el Transporte marítimo. Tesis Doctoral. Universidad Politecnica de Cataluña. España. 


\begin{tabular}{|c|c|c|}
\cline { 2 - 3 } & REVISTA SABERES APUDEP & Volumen 4 Número 1 \\
\hline
\end{tabular}

Vélez, M. (2018). Competitividad en el Sistema Portuario de Panamá. Tesis Doctoral. Institución Universitaria ESUMER. Colombia 